\title{
¿Qué libros prefieren leer niñas y niños en la etapa inicial de la enseñanza formal de la lectura?
}

\author{
What Books do Young Girls and Boys Prefer to Read \\ in the Initial Stage of Formal Reading Instruction?
}

\author{
Macarena Silva y Elvira Jéldrez \\ $\mathrm{CIAE}$, Instituto de Estudios Avanzados en Educación, Universidad de Chile
}

\section{Resumen}

\begin{abstract}
Los textos narrativos suelen ser escogidos por sobre los informativos para promover el lenguaje en las etapas iniciales de la escolaridad, con base en la creencia de que las/os niñas/os prefieren y comprenden mejor este tipo de textos. Sin embargo, la evidencia que fundamenta este principio es controvertida. Este estudio examinó la preferencia por libros de un grupo de niñas/os en dos momentos: kindergarten $(\mathrm{N}=395)$ y primero básico $(\mathrm{N}=302)$. Se le pidió a cada niño/a que revisara dos libros narrativos y dos informativos y que escogiera uno, justificando su elección. En kindergarten, las/os niños prefirieron significativamente más los libros informativos por sobre los narrativos. En kindergarten y en primero básico, se encontró una relación entre género y preferencia: los niños escogían libros informativos en mayor medida que las nińas. Las razones dadas por los escolares para escoger un libro no aludían al tipo de texto, sino al contenido, conexión con la propia experiencia, finalidad de la lectura, entre otros. Estos resultados se discuten en relación con el repertorio de libros ofrecidos a los/as niños/as para sus lecturas y la importancia del uso de textos variados en géneros discursivos y temáticas, desde las etapas de iniciación a la lectura.
\end{abstract}

Palabras claves: preferencia por libros; género de las/os niñas/os; informativos; narrativos; lectura inicial

\author{
Correspondencia a: \\ Macarena Silva \\ macarena.silva@ciae.uchile.cl \\ Periodista José Carrasco Tapia 75, Centro de Investigación Avanzada en Educación, \\ Universidad de Chile, Santiago, Chile.
}

(C) 2021 PEL, http://www.pensamientoeducativo.org - http://www.pel.cl 


\title{
Abstract
}

\begin{abstract}
Narrative texts are usually chosen rather than informational texts to promote language in the initial stages of schooling. This is based on the idea that children prefer narrative texts and that they are more appropriate for their age. However, some studies have shown that children not only understand informational texts, but also prefer them. This study examined the book preferences of a group of early readers at two times: kindergarten $(\mathrm{N}$ = 395) and first grade $(\mathrm{N}=302)$. In order to test their preferences, each child was asked to review two narrative and two informational books, choose one, and justify their choice. In kindergarten, children preferred informational books significantly more than narrative books. At both times, a significant relationship between gender and book choice was found: boys chose informational texts significantly more than girls. The reasons given by children for choosing a book are not based on the type of text, but rather on the content, theme, and visual aspects, among others. These results are discussed in relation to the book options that are provided to children for their reading activities and the relevance of offering an array of different texts in the initial stages of reading.
\end{abstract}

Keywords: book preference, gender, informational, narrative, early-readers

\section{¿Qué libros prefieren leer niñas y niños en la etapa inicial de la enseñanza formal de la lectura?}

La lectura, pero particularmente la comprensión lectora, es una habilidad clave, no solo en el ámbito académico, sino también para desempeñarse en el mundo adulto de forma competente (Cain, 2010). La lectura y la comprensión de lo que se lee son habilidades que se originan en el lenguaje oral (Catts, Fey, Zhang \& Tomblin, 1999) y que comienzan a desarrollarse de forma temprana en la interacción con otros y con materiales escritos (Whitehurst \& Lonigan, 1998). Las interacciones de lenguaje en la que participan los/as niños/as pueden ocurrir en distintas situaciones, como al recordar eventos pasados, conversaciones, al mirar programas de televisión y al compartir historias, ya sea de forma oral o utilizando libros (Skarakis-Doyle \& Dempsey, 2008). En esas interacciones de lectura, se utilizan principalmente textos y materiales narrativos (Duke, 2000; Reutzel, Smith $\&$ Fawson, 2005). Estos se han preferido porque existe la creencia de que los textos informativos pueden ser muy difíciles para escolares que se inician en la escuela (Palmer \& Stewart, 2003) y también porque se piensa que los/as niños/as prefieren los textos narrativos por sobre los de otro tipo (Mohr, 2003). Existen al menos dos consecuencias o dificultades principales que se derivan de lo anterior. La primera es que los/as niños/as estarían menos familiarizados con los textos informativos, un tipo de texto muy frecuente en la escuela (Venezky, 1995) y la segunda es que los/as niños/as se encontrarían frente a repertorios de lectura no tan diversos y que quizás no responden a todos los intereses (Duke, 2003).

Es de gran relevancia contar con textos diversos en el aula y que atiendan a los intereses de los/as niños/as, en aras de promover y mantener su motivación por la lectura, lo cual se vincula de forma directa con la dedicación de mayor tiempo a la lectura independiente y también con un mejor desarrollo de las habilidades lectoras (Chapman, Filipenko, McTavish \& Shapiro, 2007; Guthrie, Wigfield, Metsala \& Cox, 1999). Los estudios previos que han investigado las preferencias lectoras de los estudiantes tienen resultados controvertidos, pero coinciden en demostrar que los textos informativos resultan de interés para los/as niños/as que se inician en la lectura (Mohr, 2003, 2006). 
$\mathrm{Al}$ respecto, también se han estudiado las diferencias de género, para indagar si los niños y las niñas tienden a preferir algún tipo de texto por sobre otro, generalmente bajo el supuesto de que los niños preferirían textos informativos y las niñas, narrativos (Chapman et al., 1999).

Así, este estudio investiga las preferencias que tienen los/as niños/as por textos narrativos o informativos en las etapas iniciales del aprendizaje lector formal, indagando, además, si existen diferencias según el género y explorando las razones o justificaciones de sus preferencias.

\section{Lectura inicial y textos utilizados para promoverla}

La lectura compartida es una de las actividades más utilizadas para promover el lenguaje y la lectura en las etapas iniciales. La elección de libros apropiados para dicha actividad es central (Kotaman \& Tekin, 2017). Para esas interacciones, los libros que más se utilizan son los narrativos, es decir, historias o cuentos que se caracterizan por tener una estructura familiar que incluye personajes, un escenario, problema y resolución (Shapiro \& Hudson, 1991).

Si bien el uso de libros narrativos es de gran importancia para el desarrollo del lenguaje, en los últimos años se ha relevado la importancia de comprender textos informativos (Kotaman \& Tekin, 2017). Circulan diversas definiciones de este tipo de texto, pero existe consenso en plantear que lo central de un texto informativo es que transmite información sobre el mundo natural y social, generalmente dirigido desde un experto hacia alguien más novato en el tema, y que a su vez posee ciertas características lingüísticas particulares (Duke, 2003). Entre estas últimas destacan que son libros que presentan vocabulario más específico y sofisticado, sustantivos comunes genéricos en vez de propios (por ejemplo: "los delfines" en vez de "Julián el delfín") y que utilizan principalmente el tiempo presente (Yopp \& Yopp, 2012). A su vez, presentan estructuras textuales diversas, como, por ejemplo: descripciones, comparación-contraste, causa-efecto, entre otras (Meyer \& Poon, 2001). Así, para comprender textos de este tipo es importante estar familiarizado con las características presentadas, ya que se ha reportado que el conocimiento de ciertos tipos de textos y géneros discursivos no se transfiere automáticamente a otros (Duke, 2000).

Utilizar textos informativos en las etapas iniciales de la lectura es importante por distintas razones. En primer lugar, fomenta el conocimiento del mundo mediante la adquisición de información (Culatta, 2010), lo cual es central para la comprensión lectora y el aprendizaje (Cervetti \& Wright, 2020). En segundo lugar, permite acceso a un tipo de vocabulario específico y generalmente más sofisticado (Leung, 2008). Junto a lo anterior, existe también un vínculo con la motivación de los/as niños/as, ya que los libros informativos pueden satisfacer la curiosidad que muestran por los hechos del mundo natural y social (Caswell \& Duke 1998; Duke, 2003). Por su parte, la lectura comprometida, que viene dada por el interés en lo que se lee, se relaciona con mayor lectura independiente y un mejor desempeño (Williams, 2008).

Si bien la importancia de los textos informativos ha sido ampliamente documentada, aún encuentran una presencia limitada en las aulas de niveles iniciales. Por ejemplo, Pentimonti, Zucker y Justice (2011) estudiaron qué tipos de textos eran utilizados para los momentos de lectura compartida en aulas de nivel preescolar. Encontraron que, del total de lecturas, un $85,6 \%$ se realizaba con textos narrativos, mientras que solo un $5,4 \%$ con textos informativos. Hallazgos similares fueron también reportados por Yopp y Yopp (2006) extendiéndose a aulas de nivel básico. Duke (2000) observó aulas de primer grado para investigar en qué medida los/as niños/as están expuestos a este tipo de textos. En sus hallazgos, indica que se dedica en promedio solo 3,6 minutos al día a actividades que implican textos informativos y hay algunas salas que dedican 0 minuto a este tipo de actividades. A su vez, se reportó que, en las bibliotecas de aula, predominan ampliamente los textos narrativos (más de $60 \%$ de los libros disponibles) por sobre los textos informativos (con 8,2 \% de libros disponibles en promedio). Así, se reafirma que existe poca disponibilidad de libros informativos y que se utilizan en pocas instancias en la jornada escolar. 
En el caso de Chile, no existen, a nuestro conocimiento, estudios sobre el tipo de textos que se utilizan en estos niveles educativos. Como referencia de la presencia de textos informativos/narrativos, se miraron los títulos de la biblioteca digital escolar que el Ministerio de Educación chileno puso a disposición de los estudiantes. Del listado exhibido de libros para niños/as de prekinder a segundo básico, se encontraron 706 títulos, de los cuales 481 correspondían a cuentos, es decir un 68\%, mientras que el 32\% restante no contaba con una descripción específica del tipo de género discursivo, sino que solo refería a la temática, por ejemplo: astronomía, botánica, entre otros (véase https://bdescolar.mineduc.cl/).

La literatura en el tema ha establecido dos razones principales por las cuales no se incluyen suficientes textos informativos en los ańos iniciales a pesar de los beneficios que esto pudiera traer para los/as niños/as. Una primera razón se basa en la creencia de que estos textos son muy difíciles para los escolares que se encuentran en las etapas iniciales de la lectura, justamente porque presentan un vocabulario más complejo y estructuras menos familiares (Duke, 2000; Palmer \& Stewart, 2003). Esta idea ha sido refutada con evidencia que muestra que los/as niños/as de niveles iniciales sí pueden comprender textos informativos (Culatta, 2010; Williams, Stafford, Lauer, Hall \& Pollini, 2009; Williams et al., 2013) incluso a nivel similar que textos narrativos (Autor, 2017).

La segunda razón se basa en la creencia de que los/as niños/as prefieren los textos narrativos por sobre los informativos (Duke, 2000; Moss \& McDonald, 2004), ya que algunos autores plantean que los textos narrativos presentan una dificultad menor, por lo que son más accesibles para los niños en etapas iniciales y producen así un mayor disfrute (Egan, 1993). Sobre este punto existe evidencia mixta y más reducida en el caso de los/as niños/as en etapas iniciales de la lectura, pero que coincide en que sí valoran los textos informativos (Mohr, 2003; Williams, 2008).

Dada la importancia de las actividades lectoras en las etapas iniciales, es de gran relevancia conocer qué tipos de textos prefieren los/as niños/as de estas edades, especialmente considerando que este interés podría redundar en una mayor motivación por la lectura, que a su vez contribuye al desarrollo más robusto de esta habilidad.

\section{Preferencias por textos narrativos o informativos}

Barnes y Bloom (2014) ponen de relieve el hecho de que son los adultos (padres, educadores o profesores) quienes suelen elegir el material de lectura disponible para los/as nińos/as, especialmente cuando se encuentran en etapas iniciales de la lectura. Sin embargo, los adultos no necesariamente tienen un juicio preciso sobre los intereses de los/as niños/as. Por ejemplo, Beach (2015) analizó las preferencias por libros de escolares y adultos, y encontró solo un 4,36 \% de superposición en las elecciones, lo que indica que los libros escogidos por los adultos para la lectura de los/as niños/as no se condicen con los elegidos por ellos.

Un tema importante en el fomento de la motivación lectora es la posibilidad de elegir (Hudson \& Williams, 2015). De esta manera, conocer las preferencias lectoras de los estudiantes cobra especial relevancia. La literatura sobre preferencias lectoras de los/as niños/as ha exhibido resultados mixtos en cuanto a la predilección por textos narrativos o informativos. Por ejemplo, Doiron (2003), al estudiar los libros que estudiantes de 1ro a 6to grado sacaban de la biblioteca, encontró que había una predominancia de textos de ficción -donde se incluyen los narrativos-, por sobre los textos informativos - considerados de no ficción-. Barnes y Bloom (2014) evidencian que los/as niños/as tienen una preferencia por historias con personas, que hacen referencia a estados mentales y que incluyen más personajes, versus historias con objetos inanimados, con mayor referencia a acciones y con menos personajes. Los autores concluyen que existe una curiosidad en los/as nińos/as pequeños sobre las mentes de otros, que impulsa y explica el gusto por los textos de ficción. Cabe destacar que, en ambos estudios, el objetivo no era comparar o examinar la preferencia de los/as niños/as por un tipo de texto u otro, sino más bien describir las acciones lectoras, en el primer caso, y explorar elementos de la cognición social, en el otro. 
Desde una perspectiva distinta, se presenta un grupo de estudios que indaga directamente en la preferencia por tipos de textos. Así, Chapman y sus colegas (2007) estudiaron las preferencias de un grupo de escolares de primer grado utilizando dos tareas, en las cuales los/as nińos/as debían escoger entre textos, algunos narrativos y otros informativos. Los resultados mostraron que, en una tarea de elección forzada, los narrativos fueron escogidos un mayor número de veces que los informativos (68\% versus $32 \%$ de las preferencias).

Distintos fueron los hallazgos de Mohr $(2003,2006)$, quien también realizó un estudio para explorar las preferencias por textos en escolares de primer grado. Se les presentó a los/as niños/as una serie de nueve libros de calidad de distintos tipos (informativo, poesía, cuento, biografía, entre otros) de entre los cuales debían elegir uno. De los 190 participantes, 84\% escogió un libro de no ficción. El más escogido fue un texto informativo (46\% de las preferencias). Utilizando tareas similares, Kraemer, McCabe y Sinatra (2012) también encontraron una preferencia por textos informativos por sobre los narrativos en estudiantes de primer grado, lo cual se replica en niños/as de nivel parvulario (4-5 años) en los hallazgos de Kotaman y Tekin (2017).

A su vez, Williams (2008) le pidió a un grupo de estudiantes de entre 8 y 12 años que eligieran 15 libros de los exhibidos en una feria del libro. Los textos más escogidos fueron los de sagas y los relacionados con los medios (por ejemplo, libros vinculados con algún programa de televisión).

En suma, algunos estudios muestran una mayor preferencia por narrativos y sorprendentemente también aparece una preferencia por informativos incluso en nińos/as de primer grado y parvulario. Las preferencias de niños/as en este momento clave del aprendizaje lector serán investigadas en el este artículo.

\section{Preferencias y género de los/as niños/as}

En la mayoría de los artículos previamente citados se investiga a su vez la relación entre la preferencia por textos y el género de los/as niños/as. Al respecto, se ha planteado que existen ciertos estereotipos sobre qué tipo de libros son más apropiados para niños o para niñas (Chapman et al., 2007; McGeown, 2015), los cuales limitarían de cierta manera el tipo de libro que escogen o al que están expuesto los/as niños/as. Yopp y Yopp (2006) estudiaron los tipos de libros que se les leían a los/as niños/as en el hogar y en la escuela. En ambos entornos se encontró que había poca exposición a textos informativos y, junto con eso, que se les leía significativamente más libros informativos a los niños que a las niñas en el hogar. Las autoras delinean dos posibles interpretaciones: por una parte, puede ser que los adultos sigan los estereotipos planteados y, por otra, puede ser que los nińos prefieran textos informativos y que eso influencie la elección de libros que hacen los padres.

Sobre este último punto, existe un conjunto de estudios que ha investigado las preferencias lectoras de niños/ as en edad escolar y que reportan resultados no convergentes. Por ejemplo, Doiron (2003) encontró que nińos y niñas preferían textos de ficción; sin embargo, había una diferencia en la magnitud de la preferencia: las niñas escogían 3 veces más textos de ficción (narrativos) por sobre libros informativos, mientras que los niños escogían 1,3 veces más textos narrativos por sobre informativos. A su vez, Chapman y sus colegas (2007) no encontraron diferencias entre niñas y niños en cuanto a la elección de libros informativos y narrativos.

En el caso de Mohr $(2003,2006)$, si bien niños y niñas prefirieron libros de no ficción (donde se incluyen los informativos), el porcentaje varió significativamente según el género. La preferencia por este tipo de libro fue más marcada en los niños que en las niñas. Williams (2008) reporta una preferencia de ambos géneros por las series. Al comparar textos narrativos e informativos, encontró una pequeña diferencia entre niños y niñas que no alcanza a ser significativa, según la cual los niños escogen más textos de ficción y las niñas más textos de no ficción.

Así, los resultados sobre la relación entre género de los/as niños/as y preferencias lectoras de textos narrativos e informativos no son concluyentes, y será investigado en este estudio. 


\section{Razones para preferir un libro por sobre otro en los niveles iniciales de la lectura}

Dada la importancia de las preferencias lectoras, resulta relevante conocer qué aspectos consideran los escolares al elegir un libro. Chapman y sus colegas (2007) indagaron en las razones y justificaciones que daban los/as niños/as sobre su elección. Para niños y niñas fue importante el tema del libro a la hora de elegir, junto con el reconocimiento del libro (libros que habían recibidos premios), la inclusión de elementos específicos (por ejemplo: tiene muchas chinitas) y características visuales atractivas. El tipo de texto no emergió entre las razones dadas por los/as nińos/as para explicar sus preferencias. En los estudios de Mohr (2003, 2006), de entre los participantes, la mayoría de nińos y niñas basó su elección en el contenido/tema del libro. Los animales y el humor fueron los temas más valorados. En segundo lugar, justificaron su elección refiriéndose a las características de los textos, es decir, a sus atributos visibles, como la portada o las ilustraciones. Muy pocos niños/as $(n=5)$ mencionaron el tipo de texto presentado como razón para su elección (por ejemplo, por ser un texto informativo). Por su parte, Kotaman y Tekin (2017) reportaron que los/as niños/as que habían escogido textos informativos solían basar su elección en el tema del libro, mientras que quienes habían escogido textos narrativos, fundaban su preferencia en los personajes.

Dado que el tipo de texto aparece con poca notoriedad en las razones para escoger libros, en este estudio, además de indagar en los fundamentos para preferir un texto, se explorará el conocimiento de los/as niños/ as del tipo de texto del libro escogido.

\section{El estudio}

Conocer las preferencias de los/as niños/as puede ayudar a ofrecerles una selección de libros pertinentes, atendiendo a los distintos intereses. Esto puede contribuir a fomentar su vinculación y motivación hacia la lectura desde etapas iniciales y así favorecer su aprendizaje (Baker et al., 2011).

En este estudio se examina qué libros prefieren los/as niños/as, indagando a su vez si existe alguna relación entre el género de las/os participantes y su elección, junto con explorar las razones de sus preferencias. Este estudio es novedoso, ya que, además de generar evidencia nacional, los temas referidos se estudian en la etapa de iniciación a la lectura, incorporando a su vez un componente longitudinal que permitiría investigar las mencionadas variables en un momento crítico del aprendizaje lector. Las preguntas de investigación e hipótesis son las siguientes:

1. ¿Qué tipo de libro prefieren los/as niños/as en etapas de iniciación a la lectura? Basado en los hallazgos previos reportados (Mohr, 2003, 2006) se espera que los libros informativos sean escogidos por los/as niños/as en igual o mayor medida que los narrativos en kindergarten y primero básico.

2. ¿Cómo se relaciona la elección de los/as niños/as con su género? Se espera que exista una relación entre género y preferencia por cierto tipo de libros en kindergarten y primero básico. De forma más específica, se hipotetiza que los niños elegirán textos informativos por sobre los narrativos, sin embargo, no se aventura una hipótesis específica en el caso de las niñas.

3. ¿Cuáles son las razones para escoger un tipo de libro dada por los/as niños/as? No existe una hipótesis específica para esta pregunta, ya que es de carácter exploratorio y descriptivo. Sin embargo, se espera que las razones de las preferencias sean distintas entre kindergarten y primero básico, dada la mayor exposición a la enseñanza formal de la lectura que podría redundar en mayor conocimiento sobre los tipos de libros y sus características. 


\section{Método}

\section{Participantes}

Se reclutaron 395 niños/as de 5 y 6 años provenientes de 13 escuelas chilenas que participaron en el estudio. Todos los niños y niñas hablaban español como primera lengua y provenían de aulas de kindergarten -segundo nivel de transición-al comenzar el estudio (188 niñas, $M=71,98$ meses, $D E=3,94$ meses). Un año después, 302 niñas y niños de la muestra original fueron evaluados nuevamente. Las escuelas participantes fueron seleccionadas de forma aleatoria desde la lista de instituciones disponible en el sitio web del Ministerio de Educación de Chile. En el caso chileno, las escuelas tienen distintas fuentes de financiamiento: privada, particular subvencionada y pública, lo cual se vincula al nivel socioeconómico (Bellei, 2007). En este estudio se seleccionaron escuelas según su tipo de financiamiento, de forma proporcional, emulando la distribución reportada en Santiago de Chile. Así, en la muestra dos escuelas eran privadas, siete particulares subvencionadas y cuatro públicas (ver tabla 1).

Tabla 1

Descripción de la muestra por tipo de escuela

Fuente de

financiamiento de escuelas

\begin{tabular}{cccccccc}
\hline & No escuelas & Niños & Niñas & Total & Niños & Niñas & Total \\
\hline Público & 4 & 55 & 51 & 106 & 36 & 31 & 67 \\
\hline Particular subvencionado & 7 & 139 & 132 & 271 & 109 & 111 & 220 \\
\hline Privado & 2 & 13 & 5 & 18 & 11 & 4 & 15 \\
\hline Total & 13 & 207 & 188 & 395 & 156 & 146 & 302 \\
\hline
\end{tabular}

Fuente: Elaboración propia.

Dado que la experiencia con libros era relevante en este estudio, se realizó también una observación de las aulas participantes, utilizando una subescala de la pauta Early Language and Literacy Classroom Observation (ELLCO) (Smith, Brady \& Anastasopoulos, 2008). En dicha prueba se testearon ocho aspectos del ambiente letrado de las aulas: área separada; cómoda y atractiva; estado y número de libros; uso de los libros; variedad de temas; varios grados de dificultad; múltiples géneros discursivos y diversa representación. Cada aspecto es evaluado en una escala de 1 a 5 , donde 5 es ejemplar y 1 es deficiente. La moda obtenida en las aulas participantes fue de 3 o más en todos los aspectos, exceptuando los dos primeros, es decir, que existiera un área separada de libros y que ésta fuera cómoda y atractiva. Esto se debe a que algunas aulas tenían menor espacio y una infraestructura de menor calidad. Con todo, para este estudio, las variables más relevantes de esta escala se encuentran en un nivel similar que es básico o adecuado.

\section{Diseño y materiales}

El estudio es de tipo descriptivo con alcance correlacional. Considera además dos mediciones en el tiempo, una a fines de kindergarten y una a fines de primero básico. En ambos momentos, los/as niños/as completaron la tarea de preferencias y otras mediciones como parte de un proyecto más extenso. Las últimas no son parte de las preguntas de este estudio, por lo que no se reportan. A continuación, se describe la tarea de preferencias y los pasos para su construcción. 


\section{Tarea de preferencia por libros}

La construcción de la tarea de preferencia por libros se basa en los trabajos de Mohr $(2003,2006)$.

Selección de libros. Para seleccionar los libros a utilizar en la tarea de preferencias, se les pidió a siete ${ }^{1}$ expertos o conocedores de literatura infantil que crearan una lista de libros siguiendo las siguientes instrucciones: incluir libros de calidad, de distintos géneros discursivos, adecuados para niñas y niños de kindergarten y primero básico.

Los listados fueron revisados por el equipo de investigación. Se eliminaron los libros que estuvieran presentes en el plan lector chileno, libros de autores que estuvieran repetidos y los que fueron publicados antes de 2014. Lo anterior, con la intención de contar con una lista novedosa de libros que no fueran familiares para los/as niños/as.

Luego de la revisión se seleccionaron siete libros: dos informativos, dos narrativos, dos de poesía y uno mixto, que combinaba elementos narrativos e informativos.

Pilotaje. Se invitó a participar a tres escuelas para la fase de pilotaje y a un total de 30 niños/as. Luego de testear la tarea, se descartaron tres de los siete libros preseleccionados (dos poéticos y el de género mixto) dado que no fueron escogidos por los/as niños/as en ninguna oportunidad y además permitía reducir el tiempo de duración de la tarea. Todos los textos contaban con ilustraciones de calidad y eran de tamańo similar. Los textos seleccionados se muestran en la tabla 2.

Tabla 2

Descripción de libros utilizados en la tarea de preferencias

\begin{tabular}{cccc} 
Tipo de libro & Título & Autor/a & Descripción \\
\hline \multirow{2}{*}{ Informativo } & Inventario de los mares & $\begin{array}{c}\text { Emmanuelle } \\
\text { Thcoukriel }\end{array}$ & Ilustra y describe animales marinos. \\
\cline { 2 - 4 } & $\begin{array}{c}\text { Esta es la pequeña historia } \\
\text { del ferrocarril en Chile }\end{array}$ & Bárbara Cáceres & Relata la historia del ferrocarril en Chile. \\
\cline { 2 - 4 } Narrativo & El monstruo del sueño & $\begin{array}{c}\text { María Vago y Anna } \\
\text { Laura Cantone }\end{array}$ & $\begin{array}{c}\text { Cuenta la historia de un monstruo que } \\
\text { aparece en los sueños. }\end{array}$ \\
\cline { 2 - 4 } & El perro negro & Levi Pinfold & $\begin{array}{c}\text { Cuenta la historia de un perro negro que } \\
\text { atemoriza a una familia. }\end{array}$ \\
\hline
\end{tabular}

Fuente: Elaboración propia.

Preferencia. En una evaluación individual, la examinadora le presenta al niño/a los cuatro libros antes mencionados dispuestos sobre la mesa. Luego, se le pide al niño/a que los mire e inspeccione y que elija el que más le guste, elección que será registrada por la evaluadora. Esta tarea no incluye la lectura de los libros, se trata de una revisión general de los textos, pensando en la etapa previa a la lectura que influiría en la elección de un libro por sobre otro. En ambas mediciones (fines de kindergarten y fines de primero básico) se usó la misma tarea, basada en los trabajos de Mohr $(2003,2006)$.

Breve entrevista. Luego de la selección, a cada niño/a se le preguntó por las razones de su elección. La información fue elicitada con las siguientes preguntas: ¿Por qué elegiste este libro? ¿Qué tipo de libro es? (Si no responde: ¿Es una historia / cuento o un libro informativo?).

1. Los participantes fueron una educadora de párvulos con vasta experiencia en el aula, una profesora con un magíster en escritura y literatura, una bibliotecaria de biblioteca infantil municipal, dos vendedores de librerías populares, una doctora en educación infantil y una formadora de educadoras de párvulos en los cursos de lenguaje. 
Cada escuela fue contactada para participar de un estudio longitudinal sobre comprensión lectora inicial que incluía, entre otras evaluaciones, las preferencias por libros. Luego de obtener la autorización de el/la directora/a del establecimiento y contar con el consentimiento de la educadora, se envió una carta de consentimiento informado a todos los padres/cuidadores de los/as nińos/as de kindergarten (segundo nivel de transición). A los/ as niños/as cuyos padres dieron su consentimiento, se les pidió su asentimiento verbal para participar en tres sesiones individuales de evaluación con una examinadora entrenada, en una de las cuales se administró la tarea de preferencia por textos antes descrita. La sesión tuvo una duración aproximada de 15 minutos.

\section{Resultados}

Los resultados se presentan en tres secciones, atendiendo a las preguntas de investigación. En primer lugar, se presentan los hallazgos sobre preferencias, seguidos de la relación entre género de las/os participantes y preferencias y, finalmente, se reportan las razones dadas por los/as niños/as sobre su elección. En todos los análisis, los datos de los/as niños/as son tomados en su conjunto, dado que no se encontraron asociaciones entre el nivel socioeconómico de los establecimientos y la preferencia reportada $(x 2(2)=3,98, p=, 14)$.

\section{¿Qué tipo de libro prefieren los/as niños/as en etapas de iniciación a la lectura?}

El objetivo de esta pregunta es determinar si los/as niños /as tienen una preferencia por los textos narrativos o informativos que fueron presentados en kindergarten y primero básico. La frecuencia total con que se escogió un texto u otro en ambos tiempos se reporta en la tabla 3.

Tabla 3

Frecuencias para preferencias por texto según género en los tiempos 1 y 2

\begin{tabular}{ccccccccc}
\multirow{2}{*}{ Género } & \multicolumn{4}{c}{ Tiempo 1 } & \multicolumn{2}{c}{ Tiempo 2 } \\
\cline { 2 - 10 } & \multicolumn{2}{c}{ Informativo } & \multicolumn{2}{c}{ Narrativo } & \multicolumn{2}{c}{ Informativo } & \multicolumn{2}{c}{ Narrativo } \\
\hline & $\mathrm{n}$ & $\%$ & $\mathrm{n}$ & $\%$ & $\mathrm{n}$ & $\%$ & $\mathrm{n}$ & $\%$ \\
\hline Niños & 140 & 68,6 & 64 & 31,4 & 88 & 56,4 & 68 & 43,6 \\
\hline Niñas & 86 & 45,7 & 102 & 54,3 & 56 & 38,4 & 90 & 61,6 \\
\hline Total & 226 & 57,7 & 166 & 42,3 & 144 & 47,7 & 158 & 52,3 \\
\hline
\end{tabular}

Nota: N Tiempo $1=392 ;$ N Tiempo $2=302$

Fuente: Elaboración propia.

Al testear la preferencia por textos, se encontró que en kindergarten los/as niños/as prefieren significativamente los libros informativos por sobre los narrativos, $x 2(1)=9,18, p=, 002$, mientras que en primero básico no hay una diferencia significativa en la preferencia según el tipo de texto, $x 2(1)=, 65, p=, 42$.

$\mathrm{Al}$ examinar cada libro presentado en kindergarten, se encontró que la diferencia en las preferencias era estadísticamente significativa, $x 2(3)=65,10, p<, 001$. En específico, el libro más escogido fue Inventario de los mares $(\mathrm{n}=165)$, seguido por El perro negro $(\mathrm{n}=89)$, luego Esta es la pequeña historia del ferrocarril en Chile $(\mathrm{n}=77) \mathrm{y}$, finalmente, El monstruo del sueño $(\mathrm{n}=61)$. En primero básico, al examinar las diferencias en las preferencias 
por cada libro, se encontró una diferencia significativa, $x 2(3)=60,44, p<, 001$. El libro más escogido continuó siendo Inventario de los mares $(\mathrm{n}=113)$, pero la segunda preferencia fue ahora El monstruo del sueño $(\mathrm{n}=103)$, seguido por El perro negro $(\mathrm{n}=55)$ y, finalmente, Esta es la pequeña historia del ferrocarril en Chile $(\mathrm{n}=31)$.

\section{¿Cómo se relaciona la elección de los/as niños/as con su género?}

La frecuencia con la que fueron escogidos textos narrativos e informativos según el género de los/as niños/as, y en ambos tiempos, se muestra en la tabla 3. Al explorar la preferencia por tipo de texto según el género de los niños, en el tiempo 1 se encontró que existe una asociación significativa entre género y preferencia por textos informativos o narrativos, $x 2(2)=22,98, p<, 001$. Basado en las odds ratio, las odds (razón de chances) de que los niños escojan un libro informativo son 2,6 veces más altas que las de las niñas cuando están en kindergarten.

En el tiempo 2, existe también una asociación significativa entre género y preferencia, $x 2(2)=9,85, p<, 002$. Las odds (razón de chances) de que los niños escojan un libro informativo en primero básico por sobre uno narrativo, son 2,08 veces más altas que las de las niñas.

$\mathrm{Al}$ analizar la preferencia por cada tipo de libro, se encontró una diferencia significativa según el género en kindergarten, $x 2(3)=25,78, p<, 001$ y en primero básico, $x 2(3)=12,79, p=, 005$. Al explorar los residuos estandarizados, se encontró que en kindergarten la diferencia entre niñas y niños estuvo dada por el libro El perro negro, el cual fue significativamente menos escogido por niños y más escogido por niñas. En primero básico, la magnitud de las diferencias de elección de cada libro por niños y niñas fueron similares para cada libro. Más específicamente, el libro Inventario de los mares fue más escogido por niños que por niñas. La sitaución inversa fue reportada en el caso del libro El perro negro. Los otros dos textos tuvieron elecciones más parejas entre niños y niñas en primero básico.

\section{¿Cuáles son las razones para escoger un tipo de libro dada por los/as niños/as?}

Para analizar las razones que dieron las niñas y los niños participantes para justificar la elección del libro, se generaron categorías a partir de las respuestas dadas por los/as niños/as y según lo expuesto en la literatura, las cuales se presentan en la tabla 4.

Tabla 4

Categorias de respuestas sobre las razones de su elección

Categoría

Descripción

\begin{tabular}{cc}
\hline No sabe/no responde & No contesta la pregunta o declara no saber por qué eligió el texto. \\
\hline Contenido/tema & Se refiere al tema del libro o a elementos generales de su contenido. \\
Por ejemplo: 'Porque es de animales'.
\end{tabular}

\begin{tabular}{cc}
\hline Finalidad & Explicita un propósito asociado a la lectura del libro escogido. Por ejemplo: 'Para aprender'. \\
Conexión & Relata algún elemento que asocia el libro escogido con vivencias, gustos o situaciones \\
personales. Por ejemplo: 'Porque yo también tengo un perro'.
\end{tabular}

Elemento específico

Detalla algún elemento particular del libro escogido, que no refiere a su temática general. Por ejemplo: 'Porque hay un erizo'.

Característica del texto

Se refiere a elementos específicos de la apariencia del libro. Por ejemplo: 'es nuevo y lindo' o también infiere a partir de sus características: 'se ve interesante'.

\begin{tabular}{cc}
\hline Relato & El/la niño/a comienza a relatar el cuento o a describir los contenidos del texto. \\
\hline Respuesta vaga & Emite una respuesta que no puede ser clasificada en ninguna categoría dada su amplitud. \\
Por ejemplo: 'Me gusta porque sí'.
\end{tabular}

Fuente: Elaboración propia. 
Se calcularon porcentajes en base a la frecuencia con que se dio cada respuesta, lo cual se muestra en la tabla 5 . En el tiempo 1 y tiempo 2 la referencia al contenido/tema es la razón más frecuente. Sin embargo, en el tiempo 2 las respuestas están más distribuidas y aparecen también como justificaciones frecuentes la conexión con las propias experiencias y la finalidad de la lectura. En ambos tiempos, la proporción es muy similar en lo referido a elementos específicos del texto y características del texto. Entre un año y otro, disminuyen de forma importante las respuestas vagas.

Tabla 5

Razones (en porcentaje) para elegir un libro en los tiempos 1 y 2

\begin{tabular}{ccc} 
Categorías & Tiempo 1 (\%) & Tiempo 2 (\%) \\
\hline No sabe/no responde & 2,6 & 2,1 \\
\hline Contenido/tema & 37,3 & 25,3 \\
\hline Finalidad & 5,9 & 17,3 \\
\hline Conexión & 10,5 & 18,3 \\
\hline Elemento específico & 13,4 & 13,8 \\
\hline Característica del texto & 5,4 & 6,6 \\
\hline Relato & 1,8 & 2,1 \\
\hline Respuesta vaga & 23,1 & 14,5 \\
\hline
\end{tabular}

Fuente: Elaboración propia.

Para profundizar en las razones de la elección, se realizó un análisis para testear si existía alguna relación entre el libro escogido y la razón dada para escogerlo. Los resultados mostraron que sí existe una relación significativa en kindergarten y primero básico, $x 2(21)=276,49, p<, 001$ y $x 2(21)=169,93, p<, 001$. La mayoría de los/as niños/as que escogió el libro Inventario de los mares justificó su elección por el contenido del libro. En el caso del libro El monstruo del sueño, la principal razón aludía a un elemento específico del cuento. En relación con el libro Breve historia del ferrocarril en Chile, los/as niños/as respondieron principalmente haciendo un relato o descripción del texto. Finalmente, en el caso del libro El perro negro, lo fundamentan refiriendo a alguna conexión con el cuento o a un elemento específico.

Como se desprende de las categorías de respuesta, los/as niños/as no se refirieron al tipo de texto para fundamentar sus elecciones. Ante la pregunta ¿Qué tipo de libro es? un 46,3\% de los/as niños/as de kindergarten no contestó de forma correcta la pregunta sobre si el texto escogido era narrativo o informativo, cifra que se redujo a $25 \%$ en primero básico, diferencia que es marginalmente significativa, $x 2(1)=3,80 p=, 051$. Esto indica que los/as niños/as aumentan su conocimiento del tipo de texto entre kindergarten y primero básico.

\section{Discusión}

Este estudio analizó las preferencias por libros narrativos o informativos de una muestra de niños/as que se inician en el aprendizaje formal de la lectura. Los principales resultados muestran que, entre los libros presentados, existe una preferencia por textos informativos por sobre los narrativos que es estadísticamente significativa en kindergarten, mientras que en primero básico la preferencia por ambos tipos de libros es balanceada. Al incorporar como variable el género de las/os participantes, emerge una relación significativa entre género y preferencia. Así, los niños son quienes muestran una preferencia más marcada por los libros informativos en comparación con las niñas. 
Finalmente, se aprecia que el contenido o tema del libro es central para justificar las elecciones de los/as niños/ as, principalmente en kindergarten. A su vez, la conexión con las propias experiencias y la finalidad de la lectura emergen como temas relevantes para fundamentar la elección en primero básico. Es de relevancia que los/as niños/ as no mencionan el tipo de texto como razón para justificar sus elecciones, lo que pone en discusión si el tipo de texto efectivamente guía la elección. Junto a lo anterior, se reportó que a nivel de educación parvularia, cuando los/as niños/as están en kindergarten, tienen menor conocimiento del tipo de texto que están escogiendo, lo cual cambia en primero básico de forma importante. Estos hallazgos y sus implicancias se discuten a continuación.

El primer hallazgo es de gran relevancia, ya que muestra que los textos informativos sí son de preferencia de los/as niños/as, más aún, lo son por sobre los textos narrativos en kindergarten. Lo anterior está en sintonía con lo reportado por Mohr $(2003,2006)$ y Kotaman y Tekin (2012), quienes, utilizando una metodología similar, encontraron también una preferencia por textos informativos en primer grado y kindergarten, respectivamente.

Una de las principales razones que se han esgrimido para el reducido número de libros informativos presentes en las aulas de los niveles iniciales es que los/as niños/as no tienen interés por este tipo de libros a esta edad, sino que más bien prefieren los libros de ficción, especialmente los narrativos (Duke, 2003). Los resultados de este estudio robustecen la evidencia ya reportada y sugieren que es importante utilizar textos informativos junto a textos narrativos en los niveles iniciales.

La utilización de textos diversos desde los inicios del aprendizaje de la lectura podría tener efectos en la motivación por la lectura, ya que la posibilidad de elegir se relaciona con un mayor compromiso con el material leído y, a su vez, se vincula con mayor frecuencia de lectura independiente (Hudson \& Williams, 2013).

Cabe destacar que el rol de los textos narrativos es también muy importante. Estos fueron escogidos con una frecuencia levemente mayor que los informativos en primero básico. En su conjunto, los resultados muestran que tanto los libros narrativos como los informativos deben formar parte del repertorio disponible para los/as niños /as que se están iniciando en la lectura, si se quiere atender a sus intereses. La diversidad de textos puede permitir, a su vez, dar la oportunidad a los/as niños/as de explorar distintos tipos de textos, temas y géneros discursivos, enriqueciendo su experiencia lectora y, por tanto, su aprendizaje (Duke, 2003).

No de menor importancia es el hecho de atender a las preferencias de los/as niños/as de forma directa, especialmente considerando que los estudios que han investigado la superposición entre los libros que adultos reportan como interesantes para los/as niños/as y el reporte directo de los/as mismos/as muestran poca concordancia (Beach, 2015).

En relación con las diferencias de género, si bien se aprecia que los niños eligen textos informativos en mayor medida que las niñas en ambos tiempos, es relevante destacar que un número importante de niñas también prefiere textos informativos ( $45 \%$ en kindergarten y $38 \%$ en primero básico). Lo anterior es importante, ya que circulan estereotipos sobre la lectura que dicen relación con que las niñas leen más y que prefieren textos narrativos, mientras que los niños leen menos y prefieren textos informativos (McGeown, 2015). La existencia de estereotipos limita las opciones de explorar otros tipos de textos que se alejan de lo que socialmente se espera que lean niños y niñas. Según se ha reportado, estas limitaciones son más determinantes en el caso de los niños (Dutro, 2002). Una alternativa que puede contribuir a reducir estereotipos sería ofrecer amplitud de libros, especialmente si se hace desde edades tempranas donde aún no se han arraigado las creencias sobre lectura y género de niñas y niños que podrían impactar su comportamiento lector. Otra mirada, planteada por McGeown (2015), sería ofrecer a los niños más libros que respondan a sus intereses específicos, dado que son menos proclives a explorar libros que se escapan de los límites de género.

En relación con las razones esgrimidas por los/as niños/as para elegir un libro, el contenido apareció como un elemento central, tanto en kindergarten como en primero básico, lo cual se encuentra en la línea de estudios previos que muestran que el contenido y tema es la razón central para la elección de libros en los/as niños/as 
(Mohr, 2006). La conexión con elementos personales aparece como otro factor importante en las elecciones, indicando que algunos/as niños/as valoran la conexión que puede establecerse entre el material de lectura y sus propias vivencias, especialmente en primero básico. La mención a elementos específicos tiene un rol similar en ambas mediciones y la importancia de la finalidad de la lectura emerge como una razón para elegir un libro con mayor notoriedad en primero básico.

Es relevante recalcar que existe una relación entre la elección de ciertos libros y la justificación dada. De entre estos resultados es de interés que, si bien el libro Inventario de los mares y El perro negro tienen un contenido o tema similar (vinculado a animales), las razones para escoger uno u otro fueron divergentes. El primer texto mencionado fue elegido principalmente por su contenido, mientras que el segundo lo fue por la conexión con las propias experiencias. Esto podría sugerir que otros factores, además del tema, son importantes para la elección, como, por ejemplo, el tipo de texto. Es decir, si bien en ambos el tema central refiere a animales, la forma de presentar el tema, ya sea informando o narrando, puede tal vez apelar a distintos intereses y preferencias del lector, lo cual podría ser explorado en futuras investigaciones.

Cabe destacar que los/as nińos/as no mencionaron el tipo de texto como un elemento a considerar para elegir un libro. En relación con lo anterior, los resultados mostraron que los/as nińos/as de primero básico tienen un mayor conocimiento explícito sobre el tipo de texto escogido cuando se les pregunta directamente. Se especula que mayor experiencia en la enseñanza formal de la lectura puede contribuir a este conocimiento. A su vez, el conocimiento de tipos de textos narrativos e informativos no es un objetivo de aprendizaje incluido en las bases curriculares para los/as niños/as de NT2, donde solo se declara un objetivo relacionado con la comprensión oral de textos literarios y no literarios (Ministerio de Educación, 2018). Este punto es de relevancia, ya que estudios previos muestran que existe una relación entre la conciencia y conocimiento de distintas estructuras de textos (por ejemplo, incluyendo textos narrativos y variados tipos de informativos) y la comprensión lectora (Pyle et al., 2017), lo cual indica que podría ser de relevancia explicitar los elementos clave que organizan los textos desde las etapas iniciales de vinculación con la lectura.

En su conjunto, los resultados tienen importantes implicancias educativas. En primer lugar, dada la preferencia de los/as niños/as por textos informativos de entre los que les fueron presentados, en mayor medida o igual que los textos narrativos, sería importante ampliar el repertorio de libros al que están expuestos en bibliotecas escolares y de aula, así como también en los textos que se utilizan para actividades pedagógicas. La familiarización con textos informativos podría no solo atender al interés reportado de las/os niños/as, sino también tener ventajas educativas, al permitirles conocer y comprender textos con estructuras más diversas, los cuales encontrarán en abundancia durante la educación básica (Moss, 2005).

De cierta manera, y como proponen algunos autores (Duke, 2003; Yopp \& Yopp, 2000), conocer y comprender textos informativos puede ayudar a que no se produzca el llamado bajón de cuarto grado (fourth grade slump), que se refiere a las dificultades repentinas en el desempeño lector observadas en niños/as de alrededor de 9 años (Chall, 1983). Este declive ha sido asociado con el cambio en el tipo de textos a los que los/as niños/as se ven enfrentados, ya que el foco ya no estaría en aprender a leer sino en leer para aprender, lo cual se representa en el uso de un número importante de textos informativos a partir de este nivel escolar (Yopp \& Yopp, 2000). Autores como Cervetti y Wright (2020) han apuntado a que no existe ese antes/después en el desarrollo lector, sino más bien el aprendizaje de la lectura y el leer para aprender son y deberían ser procesos recíprocos, dado que no existe evidencia que induzca a retrasar o dilatar el aprendizaje a través de la lectura inicial.

De forma más explícita, comenzar a utilizar textos informativos en etapas iniciales se vincula con desarrollar un conocimiento del mundo más amplio, lo cual es clave para el desarrollo de la comprensión lectora (Barnes, Dennis \& Haefele Kalvaitis, 1996) y del aprendizaje en distintas disciplinas (Cervetti \& Wright, 2020). 
En suma, incluir un repertorio variado de tipos de textos, incluyendo temáticas diversas en la etapa de iniciación en la lectura, permitiría no solo atender a las preferencias reportadas de los/as niños/as, que consideran de forma importante los textos informativos, sino también constituiría un apoyo para su aprendizaje futuro.

Este estudio cuenta con ciertas limitaciones que es importante notar. Entre ellas, el no contar con información sobre las prácticas de aula de las que provenían los/as niños/as participantes, ya que pueden resultar importantes a la hora de la familiaridad -o no- con ciertos tipos de textos. A su vez, se incluyeron textos informativos o narrativos auténticos, que tenían temáticas distintas, lo que supone menos control en la tarea y puede influir en que la preferencia por cierto contenido esté superpuesto con la preferencia por un tipo de texto específico. Estas limitaciones abren la puerta para seguir explorando el tema de las preferencias lectoras de los/as niños/as, tema central para fortalecer las experiencias de lectura que enfrentan en la escuela.

Agradecimientos: Las autoras agradecen el financiamiento otorgado por ANID/PIA/Fondos Basales para Centros de Excelencia FB0003 y por ANID/Fondo Nacional de Desarrollo Científico y Tecnológico Fondecyt N¹1140887.

El artículo original fue recibido el 26 de enero de 2021 El artículo revisado fue recibido el 4 de julio de 2021 El artículo fue aceptado el 12 de julio de 2021

\section{Referencias}

Baker, L., Dreher, M., Shiplet, A., Beall, L., Voelker, A. N., Garrett, A. J. \& Finger-Elam, M. (2011). Children's comprehension of informational text: Reading, engaging, and learning. International Electronic Journal of Elementary Education, 4(1), 197-227. Recuperado de https://iejee.com/index.php/IEJEE/article/view/221

Barnes, J. L. \& Bloom, P. (2014). Children's preference for social stories. Developmental Psychology, 50(2), $498-503$. https://doi.org/10.1037/a0033613

Barnes, M. A., Dennis, M. \& Haefele Kalvaitis, J. (1996). The effects of knowledge availability and knowledge accessibility on coherence and elaborative inferencing in children from six to fifteen years of age. Journal of Experimental Child Psychology, 61(3), 216-241. https://doi.org/10.1006/jecp.1996.0015

Beach, J. (2015). Do Children Read the Children’s Literature Adults Recommend? A Comparison of Adults’ and Children's Annual “Best” Lists in the United States 1975-2005. New Review of Children's Literature and Librarianship, 21(1), 17-41. https://doi.org/10.1080/13614541.2015.976075

Bellei, C. (2007). Expansión de la educación privada y mejoramiento de la educación en Chile. Evaluación a partir de la evidencia. Pensamiento Educativo. Revista de Investigación Educacional Latinoamericana, 40(1), 285-311.

Cain, K. (2010). Reading development and difficulties: an introduction. Wiley-Blackwell.

Caswell, L. J. \& Duke, N. K. (1998). Non-narrative as a catalyst for literacy development. Language Arts, 75(2), $108-117$.

Catts, H. W., Fey, M. E., Zhang, X. \& Tomblin, J. B. (1999). Language basis of reading and reading disabilities: Evidence from a longitudinal investigation. Scientific Studies of Reading, 3(4), 331-361. https://doi.org/10.1207/s1532799xssr0304_2

Cervetti, G.N. \& Wright, T.S. (2020). The role of knowledge in understanding and learning from text. En E.B. Moje, P. Afflerbach, P. Enciso, \& N.K. Leseaux (Eds.), Handbook of reading research (Vol. 5) (pp. 237-260). Nueva York, NY: Routledge.

Chall, J.S. 1983. Stages of reading development. Nueva York, NY: McGraw- Hill. 
Chapman, M., Filipenko, M., McTavish \& Shapiro, J. (2007). First graders' preferences for narrative and/or information books and perceptions of other boys' and girls' book preferences. Canadian Journal of Education/Revue canadienne de l'éducation, 30(2), 531-553. https://doi.org/10.2307/20466649

Culatta, B., Hall-Kenyon, K. \& Black, S. (2010). Teaching expository comprehension skills in early childhood classrooms. Topics in Language Disorders, 30(4), 323-338. https://doi.org/10.1097/TLD.0b013e3181ff5a65

Doiron, R. (2003). Boy Books, Girl Books. Teacher Librarian, 30(3), 14-16.

Duke, N. K. (2000). 3.6 minutes per day: The scarcity of informational texts in first grade. Reading Research Quarterly, 35(2), 202-224. https://doi.org/10.1598/RRQ.35.2.1

Duke, N. K. (2003). Reading to learn from the very beginning: Information books in early childhood. Young Children, 58(2), 14-20.

Dutro, E. (2002). 'But that's a girls' book!': Exploring gender boundaries in children's reading practices. The Reading Teacher, 55(4), 376-384. Recuperado de https://www.jstor.org/stable/20205065

Egan, K. (1993). Narrative and learning: A voyage of implications. Linguistics and Education, 5(2), 119-126. https://doi.org/10.1016/0898-5898(93)90028-9

Guthrie, J. T., Wigfield D. A., Metsala, J. L. \& Cox, K. E. (1999). Motivational and cognitive predictors of text comprehension and reading amount. Scientific Studies of Reading, 3(3), 231-256. https://doi.org/10.1207/s1532799xssr0303_3

Hudson, A. K. \& Williams, J. A. (2015). Reading Every Single Day. The Reading Teacher, 68(7), 530-538. https://doi.org/10.1002/trtr.1349

Kotaman, H. \& Tekin, A. K. (2016). Informational and fictional books: young children's book preferences and teachers' perspectives. Early Child Development and Care, 187(3-4), 600-614. https://doi.org/10.1080/03004430.2016.1236092

Kraemer, L., McCabe, P. \& Sinatra, R. (2012). The effects of read-alouds of expository text on first graders' listening comprehension and book choice. Literacy Research and Instruction, 51(2), 165-178. https://doi.org/10.1080/19388071.2011.557471

Leung, C. B. (2008). Preschoolers' Acquisition of Scientific Vocabulary Through Repeated Read-Aloud Events, Retellings, and Hands-On Science Activities. Reading Psychology, 29(2), 165-193. https://doi.org/10.1080/02702710801964090

McGeown, S. P. (2015). Sex or gender identity? Understanding children's reading choices and motivation. Journal of Research in Reading, 38(1), 35-46. https://doi.org/10.1111/j.1467-9817.2012.01546.x

Meyer, B. J. F. \& Poon, L. W. (2001). Effects of structure training and signaling on recall of text. Journal of Educational Psychology, 93(1), 141-159. Recuperado de https://psycnet.apa.org/buy/2001-16705-012

Ministerio de Educación. (2018). Bases curriculares de la educación parvularia. Recuperado de https://parvularia.mineduc.cl/wpcontent/uploads/sites/34/2018/03/Bases_Curriculares_Ed_Parvularia_2018.pdf

Mohr, K. A. J. (2003). Children's choices: A comparison of book preferences between Hispanic and Non- Hispanic first-graders. Reading Psychology: An International Quarterly, 24(2), 163-176. https://doi.org/10.1080/02702710308231

Mohr, K. A. J. (2006). Children's choices for recreational reading: A three-part investigation of selection preferences, rationales, and processes. Journal of Literacy Research, 38(1), 81-104. https://doi.org/10.1207/s15548430jlr3801_4

Moss, G. \& McDonald, J. W. (2004). The borrowers: library records as unobtrusive measures of children's reading preferences. Journal of Research in Reading, 27(4), 401-412. https://doi.org/10.1111/j.1467-9817.2004.00242.x

Moss, B. (2005). Making a Case and a Place for Effective Content Area Literacy Instruction in the Elementary Grades. The Reading Teacher, 59(1), 46-55. https://doi.org/10.1598/RT.59.1.5

Palmer, R. G. \& Stewart, R. A. (2003). Nonfiction trade book use in primary grades. The Reading Teacher, 57(1), 38-49.

Pentimonti, J. M., Zucker, T. A. \& Justice, L. M. (2011). What are Preschool Teachers Reading in Their Classrooms? Reading Psychology, 32(3), 197-236. https://doi.org/10.1080/02702711003604484

Pyle, N., Vasquez, A. C., LignugarisKraft, B., Gillam, S. L., Reutzel, D. R., Olszewski, A., ... Pyle, D. (2017). Effects of Expository Text Structure Interventions on Comprehension: A Meta-Analysis. Reading Research Quarterly, 52(4), 469-501. https://doi.org/10.1002/rrq.179

Reutzel, D., Smith, J. \& Fawson, P. (2005). An evaluation of two approaches for teaching reading comprehension strategies in the primary years using science information texts. Early Childhood Research Quarterly, 20(3), 276-305. https:// doi.org/10.1016/j.ecresq.2005.07.002 
Shapiro, L. R. \& Hudson, J. A. (1991). Tell me a make-believe story: Coherence and cohesion in young children's picture-elicited narratives. Developmental Psychology, 27(6), 960-974.

Silva, M. (July, 2017). The skills behind early comprehension of narrative and expository texts. Presentation at the Society for the Scientific Study of Reading XXIV Annual Meeting, Halifax, Canada.

Skarakis-Doyle, E. \& Dempsey, L. (2008). Assessing Story Comprehension in Preschool Children. Topics in Language Disorders, 28(2), 131-148. https://doi.org/10.1097/01.tld.0000318934.54548.7f

Smith, M., Brady, J. \& Anastasopoulos, L. (2008). Early Language and Literacy Classroom Observation. Baltimore, CA: Brookes Publishing.

Venezky, R. L. (2000). The origins of the present-day chasm between adult literacy needs and school literacy instruction. Scientific Studies of Reading, 4(1), 19-39. https://doi.org/10.1207/S1532799XSSR0401_3

Whitehurst, G. J. \& Lonigan, C. J. (1998). Child Development and Emergent Literacy. Child Development, 69(3), 848-872. https://doi.org/10.1111/j.1467-8624.1998.tb06247.x

Williams, L. M. (2008). Book selections of economically disadvantaged black elementary students. Journal of Educational Research, 102(1), 51-63. https://doi.org/10.3200/JOER.102.1.51-64

Williams, J. P., Stafford, K. B., Lauer, K. D., Hall, K. M. \& Pollini, S. (2009). Embedding reading comprehension training in content-area instruction. Journal of Educational Psychology, 101(1), 1-20. https://doi.org/10.1037/a0013152

Williams, J.P., Pollini, S., Nubla-Kung, A., Snyder, A., García, A., Ordynans, J. \& Atkins, G. (2013). An intervention to improve comprehension of cause/effect through expository text structure instruction. Journal of Educational Psychology, 106(1), 1-17. https://doi.org/10.1037/a0033215

Yopp, R. H. \& Yopp, H. K. (2000). Sharing informational text with young children. The Reading Teacher,53(5), 410-423.

Yopp, R. H. \& Yopp, H. K. (2006). Informational text as read-alouds at school and home. Journal of Literacy Research, 38(1), 37-51. https://doi.org/10.1207/s15548430jlr3801_2

Yopp, R. H. \& Yopp, H. K. (2012). Young children's limited and narrow exposure to informational text. The Reading Teacher, 65(7), 480-490. https://doi.org/10.1002/TRTR.01072 